\title{
DON JUAN MANUEL: REALIDAD TEXTUAL Y ORIGINALIDAD CREADORA
}

\author{
Francisco Javier DÍEZ DE REVENGA \\ Universidad de Murcia
}

Se han estudiado ya en este congreso numerosos aspectos de la época del rey aragonés Jaime II, que sin duda han dilucidado muchos pormenores poco conocidos de tan interesante período de la historia medieval, que importa, y de forma decisiva a las actuales regiones de Valencia y Murcia, y que tanto tiene que ver con ciudades muy importantes de estas regiones como son la propia Murcia, Alicante, Lorca, Orihuela, Cartagena y tantos otros territorios de frontera de los reinos de Murcia, Valencia y Granada. Territorios que Don Juan Manuel, protagonista de estas páginas, conocía muy bien y tal como dejó claro en tempranas y singulares descripciones, presentes en el Libro de la caza, comenzando por Villena, la mejor zona para la caza de garzas, ánades, con halcones y con azores, perdices, codornices, flamencos, liebres y conejos. De allí, a Elda y Novelda, Vinalopó abajo, hasta llegar a la laguna de Santa Pola, rica en aves acuáticas. Luego Crevillente, Albatera, la huerta de Orihuela, cuya fragosidad dificulta el ejercicio de tan señorial deporte, como ocurre en Monteagudo y en su almarjal. Otro almarjal, el de Cartagena, será rico en aves como todo el Valle del Guadalentín y Lorca.Y don Juan Manuel se complacerá en dejarnos un relato ameno de sus aficiones deportivas y de su dominio del terreno.

Era la del nieto de San Fernando una época especialmente revuelta y todos los historiadores han sido unánimes en señalar las dificultades que asolaban estas tierras durante aquellos años cruciales. Nuestro personaje era muy especial: levantisco aristócrata, orgulloso de su estirpe real, contribuyó y mucho al desarrollo de estos territorios, pero también es muy cierto que su contribución trajo consigo muy complejos problemas. Su figura atraviesa los años de la época de Jaime II de Aragón, su suegro y protector, con quien se alió para defenderse de sus propios parientes, su sobrino Fernando IV de Castilla y su sobrino nieto Alfonso XI. Está presente también en la de 
Alfonso IV de Aragón, que le concedió el título de Príncipe de Villena, y en la de Pedro IV, que le otorgó el de Duque de Villena. La corte de Jaime II fue la que le abrevió el nombre y le dio justamente éste que ahora utilizamos y con el que todos le conocemos, como si se tratase de un nombre y un apellido modernos: Don Juan Manuel. Tal denominación, debida a la comodidad de la cancillería aragonesa, como sabemos, era impensable en Castilla, donde siempre fue nombrado tal como él mismo firmaba: Don Juan, hijo del infante Don Manuel. Traspasa del mismo modo el reinado de varios monarcas castellanos, su primo Sancho IV, el hijo de éste y el nieto (Fernando IV y Alfonso $\mathrm{XI}$ ) en cuyas minoridades ejerció su influencia e incluso la regencia en la del segundo de ellos, y en cuyas mayorías provocó disensiones y conflictos de lo más variado y diverso, sufrió persecución y a punto estuvo de ser asesinado por orden de su sobrino nieto, el rey Alfonso XI.

Para los historiadores de la literatura se nos ofrece, sin embargo, muy distinto, como un prudente escritor, embarcado en el proyecto más serio de renovación de las letras castellanas del siglo XIV, actividad en la que ocupa un lugar de honor, porque en él se ha considerado al primer escritor de nuestras letras con conciencia de serlo, al autor de una obra didáctica dechado de prudencia y sabiduría, al renovador de nuestro idioma y al creador de una prosa casi mayor de edad, capaz de expresar conceptos y sentimientos, continuadora de la labor iniciada por el tío de nuestro Príncipe y rey Alfonso $X$ el Sabio.

No me voy a referir, desde luego a su figura histórica. Otros lo han hecho y con títulos más que sobrados. Han aportado documentación inédita y han abierto caminos, desde los trazados, hace ya muchos años, por Giménez Soler, cuyo nombre siempre ha de ser recordado al comienzo de cualquier estudio sobre Don Juan Manuel'. Hemos de señalar, como complemento de las referencias bibliográficas más conocidas, que muy recientemente, en la década de los ochenta, Don Juan Manuel recibió, como personaje histórico, estadista y político, atención por parte de prestigiosos historiadores, que documentaron sus difíciles relaciones con los territorios y los concejos de las regiones fronterizas de su adelantamiento. Aurelio Pretel ${ }^{2}$, el año del centenario, 1982, dedicó un voluminoso libro al que él llamaba «el señor de la llanura» y sus relaciones con las comarcas de la actual provincia de Albacete, mientras Juan Torres Fontes, en ese mismo año del centenario, estudiaba las «tensiones y conflictos», con el concejo murciano ${ }^{3}$, que ampliaba, en otro artículo de 1986, al señorío de Cartagena ${ }^{4}$. En el libro del Centenario, que llevaron a cabo la Universidad de Murcia y la Academia

1 Andrés GIMÉNEZ SOLER, Don Juan Manuel: biografía y estudio crítico, La Académica, Zaragoza, 1932.

2 Aurelio PRETEL, Don Juan Manuel, señor de la llanura: repoblación y gobierno de la Mancha albacetense en la primera mitad del siglo XIV, Instituto de Estudios Albacetenses, Albacete, 1982.

3 Juan TORRES FONTES, «Murcia y don Juan Manuel: tensiones y conflictos", Don Juan Manuel VII Centenario, Universidad de Murcia-Academia Alfonso X el Sabio, Murcia, 1982, pp. 17-26.

4 Juan TORRES FONTES, "Don Juan Manuel señor de Cartagena", Estudios en homenaje a Don Claudio Sánchez Albornoz en sus noventa años, Facultad de Filosofía y Letras, Universidad de Buenos Aires, 1983-1986, IV, pp. 35-57. 
Alfonso $\mathrm{X}$ el Sabio, se contó con granados especialistas que abordaron otros aspectos históricos, especialmente Julio Valdeón ${ }^{5}$ y Reinaldo Ayerbe-Chaux, encargado de examinar las complejas relaciones de Don Juan Manuel con la Corona de Aragón ${ }^{6}$. A todos ellos me remito.

$Y$ me van a permitir que tampoco haga en este breve espacio temporal de que dispongo ningún panorama general sobre la cultura o la literatura de la época de Don Juan Manuel, porque está hecho en muchos lugares. Les puedo citar multitud de estudios y libros. Si quieren ustedes saber de la relación de Don Juan Manuel con estas tierras, también es un aspecto muy estudiado. Ángel Luis Molina y yo, hace ya muchísimos años, estudiamos las tierras murcianas de Don Juan Manuel a través del Libro de la caza y en la Historia de la Literatura Murciana, que publicamos Mariano de Paco y yo en $1989^{\circ}$, hablamos de la relación con Murcia de don Juan Manuel, desde el punto de vista literario. Ángel Luis Molina estudiaría también los dominios del mal llamado infante y otros estudios relacionarían con Murcia su actividad ${ }^{9}$. No voy a volver sobre cosas estudiadas, lo que constituiría una falta de respeto hacia ustedes, que no voy a cometer. Prefiero, sin embargo, y en justa compensación, hacer lo que aquel bondadoso cristiano que en vez de dar como limosna el pescado al hambriento, le daba la caña con qué pescarlo. Y mi contribución a este congreso no va a pasar de ser una caña en forma de valoración de las últimas aportaciones a la realidad textual de la obra juanmanuelina, es decir dónde y con qué confianza pueden leerse sus obras, a tenor de las últimas y más recientes aportaciones en este terreno. Pero no por ello renunciaré a hacer al final una justificada valoración literaria de nuestro Don Juan Manuel.

Prefiero, entonces, reflexionar en esta ponencia sobre los instrumentos y documentos que han mejorado nuestras posibilidades de acceso a los textos de Don Juan Manuel en los años que han transcurrido desde el Centenario hasta hoy, es decir, desde 1982 a 1996. Y hay que señalar en primer lugar que la penuria de textos de las obras juanmanuelinas antes del Centenario era alarmante. Un autor tan significativo como él no contaba con textos que pudiesen ser fiables y era una verdadera pena, que salvo la edición del Libro de Patronio o El Conde Lucanor que Blecua había hecho estupendamente para Clásicos Castalia ${ }^{10}$, ninguna de las demás obras se podían

5 Julio VALDEÓN BARUQUE, “Don Juan Manuel y Peñafiel», Don Juan Manuel VII Centenario, Unizersidad de Murcia-Academia Alfonso X el Sabio, Murcia, 1982, pp. 385-395.

6 Reinaldo AYREBE-CHAUX, «Don Juan Manuel y la Corona de Aragón. La realidad política y el ideal de los tratados", Don Juan Manuel VII Centenario, Universidad de Murcia-Academia Alfonso $X$ el Sabio, Murcia, 1982, pp. 17-26.

7 Francisco Javier DÍEZ DE REVENGA y Ángel Luis MOLINA MOLINA, «Don Juan Manuel y el reino de Murcia: notas al Libro de la caza", Miscelánea Medieval Murciana, 1, 1973, pp. 9-48.

8 Francisco Javier DÍEZ DE REVENGA y Mariano DE PACO, Historia de la Literatura Murciana, Universidad de Murcia-Academia Alfonso X el Sabio-Editora Regional de Murcia, Murcia, 1989.

9 Ángel Luis MOLINA MOLINA, "Los dominios de Don Juan Manuel», Don Juan Manue/ VII Centenario, Universidad de Murcia-Academia Alfonso X el Sabio, Murcia, 1982, pp. 215-226.

10 DON JUAN MANUEL, El Conde Lucanor, Castalia, Madrid, 2ª edición, Madrid, 1971. 
consultar con una mínima coherencia y con una imprescindible seguridad de que lo que estábamos leyendo era lo que Don Juan Manuel había escrito. Era penosa, por ejemplo, la edición del Libro de la caza, que había hecho Castro y Calvo ${ }^{11}$. Otras ediciones eran inasequibles o inencontrables. El resto de las obras de Don Juan Manuel era imposible de consultar.

Pero, por fortuna para los estudiosos de la literatura de Don Juan Manuel, el prestigioso e incansable filólogo, profesor José Manuel Blecua, dio a conocer en 1981 y 1983, por fin, su edición de las Obras completas ${ }^{12}$ del hijo del infante Don Manuel, en dos volúmenes, cuyos resultados textuales y bibliográficos hemos, hoy, de celebrar, ya que nos permiten asegurar que en los últimos años, se ha dado un gran avance en el campo de las ediciones del Príncipe de Villena. Hemos visto, casi coincidiendo con el centenario, aparecer esta edición de las Obras completas hecha por José Manuel Blecua, lo que ha supuesto, en el campo del estudio de los textos juanmanuelinos, un avance absolutamente singular. Blecua no lleva a cabo una edición al uso. Prefiere concentrar todos sus esfuerzos en cuidar al máximo el texto. $Y$ merece la pena que nos detengamos en por qué esta edición es tan valiosa. En primer lugar porque es la obra de toda una vida. Él lo explica bien en la introducción y a ella remitimos. Comenzó su trabajo en los años treinta, y, de hecho, publicó una edición del Libro infinido en 1938, en Zaragoza ${ }^{13}$. Pero, a pesar de que siempre siguió trabajando sobre los textos del ilustre autor medieval, diversos proyectos de otros investigadores le hacían desistir de trabajar en Don Juan Manuel. Pero desde los años cincuenta todo estaba abandonado, $y$, tras recuperar la tarea, se decide finalmente a editar todos los textos que no aparecen ya hasta las fechas, antes señaladas, de 1981-1983. La edición comprende un primer volumen con el Libro del Caballero y del Escudero, Libro de las armas, Libro enfenido, Libro de los estados, Tratado de la Asunción de la Virgen María y Libro de la Caza, mientras que el segundo volumen recoge El Conde Lucanor y la Crónica abreviada. Para todos los textos, Blecua se sirve del conocido manuscrito de la Biblioteca Nacional de Madrid (el 6376), mientras que para la Crónica abreviada utiliza el manuscrito de la misma Biblioteca, número 1356. En el caso de El Conde Lucanor, que, como se sabe, cuenta con otros manuscritos, especialmente señalables el Códice de Puñonrostro de la Real Academia Española y el manuscrito de la Real Academia de la Historia, Blecua ha utilizado todos ellos así como la edición de Argote de Molina.

Ver reunidos en una misma edición, admirablemente impresa, perfectamente justificada, y con un aparato crítico exhaustivo, todas las obras de Don Juan Manuel fue un gran alivio para los estudiosos de la obra literaria de este gran escritor que tanto

11 DON JUAN MANUEL, Libro de la caza, edición de José María Castro y Calvo, C.S.I.C., Barcelona, 1945.

12 DON JUAN MANUEL, Obras completas, edición de José Manuel Blecua, Gredos, Madrid, 19811983.

13 DON JUAN MANUEL, Libro Infinido. Tratado de la Asunción de la Virgen, edición de José Manuel Blecua, Universidad, Zaragoza, 1937-1938, pp. 2-28 y 165-208. Reedición en Universidad de Granada, Granada, 1952. 
veló porque su obra llegara a nosotros de la mejor forma posible. La desgracia que impidió durante siglos que esta decisión de Don Juan Manuel tuviese frutos, por fin se veía superada.

Posteriormente, un especialista antes citado, Reinaldo Ayerbe-Chaux ha publicado en 1989 una edición de Cinco tratados ${ }^{14}$, que merece también un detenido comentario.

Reinaldo Ayerbe-Chaux ha demostrado a lo largo de numerosos artículos y algún libro anterior (El Conde Lucanor. Materia tradicional y originalidad creadora ${ }^{15}$ ) su fidelidad al estudio de Don Juan Manuel y su acertada dedicación al conocimiento del magnate y escritor castellano del siglo XIV. Por ello, no nos puede extrañar la calidad, precisión, cuidado y documentación con que ha llevado a cabo la edición de algunas de las obras de este interesante autor medieval español. En concreto se trata de cinco de sus tratados: el Libro del cavallero et del escudero, el Libro de las tres razones, el Libro enfenido, el Tratado de la Asunción de la Virgen y el Libro de la caça. Cinco obras de las que existían algunas ediciones anteriores, en las que se habían venido perpetuando errores de trascripción, excepción hecha de la gran edición de José Manuel Blecua (Madrid, 1981-1983). El procedimiento utilizado por Ayerbe-Chaux para conseguir un texto depurado y evitar los errores anteriores ha consistido en trascribir directamente del famoso Ms. 6376 de la Biblioteca Nacional de Madrid sin tener en cuenta las trascripciones realizadas por otros. $Y$ una vez llevada a cabo, resueltos los habituales problemas paleográficos y establecida la puntuación, ha cotejado su texto con la que se considera la edición más respetada del noble castellano, la antes citada de José Manuel Blecua. A partir de ahí ha adoptado las lecturas de Blecua que ha considerado mejores, siempre que no se ha coincidido en las mismas, indicándolo en tales casos en el aparato de notas.

Precede a la edición un estudio preliminar muy completo, informativo y valioso, tanto sobre la figura de don Juan Manuel como sobre su obra partiendo de datos ya conocidos que van siendo enriquecidos con las últimas aportaciones de la bibliografía especializada y del propio autor de la edición. Ayerbe-Chaux es cuidadoso y honesto hasta el extremo de reconocer su error al haber designado en el pasado a don Juan Manuel con el título de «infante», que el noble castellano jamás ostentó. Ahora podría documentar tal aspecto consultando el estudio sobre el particular, que hice en colaboración con María Concepción Ruiz Abellán sobre «Denominación y títulos de Don Juan Manuel» ${ }^{16}$. De su reflexión sobre la personalidad de Don Juan Manuel interesa mucho su opinión sobre el lugar excepcional que ocupa el autor en la literatura medieval, ya que por su condición de político, de hombre de mundo que podríamos decir utilizando una expresión actual, su experiencia contaba mucho en sus obras.

14 Juan Manuel, Cinco Tratados. edición de Reinaldo Ayerbe-Chaux, The Hispanic Seminary for Medieval Studies, Madison, 1989.

15 Reinaldo AYERBE-CHAUX, El Conde Lucanor. Materia tradicional y originalidad creadora, Porrúa-Turranzas, Madrid, 1975.

16 Francisco Javier DÍEZ DE REVENGA y María Concepción RUIZ ABELLÁN, «Denominación y títulos de Don Juan Manuel», Miscelánea Medieval Murciana, 8, 1981, pp. 9-29. 
Mucho más amplio, original y completo es el estudio dedicado a cada uno de los cinco tratados. En el dedicado al Libro del cavallero et del escudero se plantea el análisis partiendo del fondo histórico que supone la caballería y del fondo literario que constituyen los libros de esta especialidad, para finalizar centrando el análisis de la obra en relación con la propia vida del autor y destacando que en la época más tormentosa de su existencia fue cuando don Juan Manuel escribió sus obras más conseguidas, como este Libro del cavallero et del escudero. En el de Las tres razones o Libro de las armas valora especialmente la mezcla de historia y leyenda y la «fluidez deliciosa» de la narración, mientras que en torno al Libro enfenido reivindica su importancia dentro de la obra de don Juan Manuel sobre todo por la relación planteada y tan interesante autor-obra-destinatario. Sobre el Tratado de la Asunçión de la Virgen valora su condición de documento histórico sobre las creencias religiosas de la época y su calidad de páginas últimas del autor, «dictadas por el corazón y por un tierno afecto a la Virgen». El estudio más extenso es el dedicado, por último, al Libro de la caza, encuadrado en los tratados de ceterería a que tan aficionados eran los nobles y grandes señores en la Edad Media. Plantea Ayerbe-Chaux en esta ocasión con todo detalle la cuestión de la autoría directa del libro, que atribuye, tras examinar todas las posibilidades y variados argumentos, a la cancillería del príncipe castellano, aunque advirtiendo que tal supuesto sólo es aplicable al Libro de la caza y no a obras más maduras, y más personales, como el Libro de los Estados y El Conde Lucanor.

Los textos de cada uno de los cinco tratados van acompañados de interesantes notas que atienden a dos aspectos: al textual, antes citado, y a la explicación de los textos con múltiples referencias de carácter histórico, literario, cultural y bibliográfico. Sin ser desmedido en la cuestión, el editor cuida atender a los aspectos más concretos de una forma muy sintética, de manera que no se produzca farragosa erudición, antes bien un material explicativo muy claro y conciso. Tal apartado informativo se completa con la presencia de un muy amplio glosario final, muy útil ante la dificultad que pudieran plantear algunas lecturas. Subrayemos, por último, las referencias bibliográficas que sobre los estudios en torno a don Juan Manuel realiza el editor. Son muy completas igualmente. La bibliografía en torno al autor, además de ser muy amplia, se halla dispersa por todas partes del mundo, ya que ha sido objeto de la atención de hispanistas de este y del otro lado del Atlántico. Ayerbe-Chaux ha incorporado a su obra la cita de trabajos a veces publicados en no muy difundidos medios publicísticos.

Se trata por tanto de una obra muy completa en todos sus aspectos que une, a todas las cualidades que hemos señalado de carácter científico, la grata y manejable presentación del volumen, que sin duda está contribuyendo, por todo ello, en gran manera a la difusión de la obra menos conocida del autor de El Conde Lucanor en los medios hispanísticos no sólo americanos. Ayerbe-Chaux une a su bibliografía juanmanuelina, muy estimada por la crítica precedente, esta cuidada edición que pone de relieve su buen hacer, su preparación y su inteligencia en el manejo de textos que tanta dificultad presentan para el estudioso y para el lector en general.

Nos vamos a referir, finalmente, a una edición, aun más reciente, de 1992-1994, que sirve para conocer muy bien algunos aspectos de la cultura escrita del siglo de 
Don Juan Manuel y pone a disposición de los lectores uno de los códices que contiene su Libro de Patronio o El Conde Lucanor. Estoy aludiendo a la edición del Códice de Puñonrostro ${ }^{17}$, que llevaron a cabo la Real Academia Española, la Universidad de Murcia y la Asamblea Regional en 1992 con un breve prólogo de José Manuel Blecua. A esta edición siguió la publicación de una edición y trascripción de este códice publicada en 1994 por la Asamblea y la Universidad, al cuidado de Juan Torres Fontes, Ángel Luis Molina Molina, Francisco Veas Arteseros y Juan Francisco Jiménez Alcá$z^{2}{ }^{18}$. El Códice de Puñonrostro como nos refieren sus editores es un valioso manuscrito que contiene, además de El Conde Lucanor, el texto del Sendebar, colección de cuentos mandada traducir por el Infante Don Fadrique, hermano de Alfonso X el Sabio, y tío de Don Juan Manuel. Se trata de uno de los textos de narrativa medieval en castellano más interesantes de todo el período desde el punto de vista del desarrollo de la narrativa en las literaturas modernas. Completan el códice varios textos más: una explicación del Padre Nuestro, el testamento de Alonso de Cuenca, físico del rey, una epístola de San Bernardo a San Ambrosio y un tratado de moral, religión y ciencias, en forma de diálogo. El códice puede fecharse a finales del siglo XIV.

Con todos estos textos, recientemente publicados, ya estamos en condiciones de leer a la figura más importante de la literatura peninsular de la primera mitad del siglo XIV junto al Arcipreste de Hita, un poeta, vinculado todavía al mester de clerecía, que nace a finales del siglo XIII, como Don Juan Manuel, y que deja un sólo libro, El libro de Buen Amor, joya literaria, muy diferente de la colección cuentística del sobrino de Alfonso $X$, pero coincidente en algunos puntos, especialmente en contener la herencia del relato breve, del cuento y del apólogo oriental, trasmitido a través de la cultura árabe, que, sin duda, es la mejor aportación juanmanuelina. Esa herencia conecta a Don Juan Manuel con el gran esplendor del cuento europeo del siglo XIV, cuyas máximas figuras son Giovanni Boccaccio con su Decameron y Geoffrey Chaucer con sus Cuentos de Canterbury.

Querría, para terminar esta intervención, hacer referencia, como he anunciado, a un aspecto estrictamente literario, que es muy representativo de la literatura ejercida por el Arcipreste de Hita y Don Juan Manuel. Y para ello, vamos a tomar como ejemplo, que comentaremos, una lectura, una lectura cualquiera, que naturalmente no vamos a hacer en este momento, pero que todos podemos recordar. Tomemos como ejemplo, uno de los apólogos más conocidos, el cuento XXXV de El Conde Lucanor, «De lo que aconteció a un mancebo que casó con una mujer muy fuerte y muy brava". Todos somos capaces de recordar ahora mismo el cuento. El Conde Lucanor pide a Patronio, como es habitual, que resuelva un problema de sus estados, en este caso el de un criado suyo que pretenden casarlo con una mujer muy rica pero también muy

17 Códice de Puñonrostro. El Conde Lucanor y otros textos medievales, edición de José Manuel Blecua, Real Academia Española-Universidad de Murcia-Asamblea Regional de Murcia, 1992.

18 Códice de Puñonrostro. El Conde Lucanor y otros textos medievales, edición y trascripción de Juan Torres Fontes, Ángel Luis Molina Molina, Francisco Veas Arteseros y Juan Francisco Jiménez Alcázar, Universidad de Murcia-Asamblea Regional de Murcia, 1994. 
brava. Patronio le cuenta para ayudarle un cuento, cuya tradición oriental se manifiesta desde el principio, el del «fijo de un hombre bueno que era moro», al que casaron con una mujer "muy fuerte y muy brava». La noche de bodas, el joven casado, va pidiendo de forma sucesiva a todos los animales de la casa que le den agua para las manos, y conforme cada uno de ellos (perro, gato y caballo) no lo hace, los va apuñalando y despedazando de la forma más sañuda que pudiera imaginarse, ante el espanto de la recién casada, que va poco a poco perdiendo su bravura. Cuando llega el momento en que le pide el agua a la esposa, ésta sin rechistar se la da, con lo que queda totalmente amansada y el mancebo triunfador. A la mañana siguiente, la familia conoce sin salir de su sorpresa lo sucedido, y, a los pocos días, intenta el padre de la novia, ya en su casa, mostrarse igualmente fiero con su esposa, y para ello mata, con similares intenciones, un gallo, pero la mujer le avisa que ya es demasiado tarde, "ca ya bien nos conocemos». «Et porque don Johan lo tuvo por buen enxiemplo, fízolo escribir en este libro et fizo estos versos que dicen así»:

Si al comienzo no muestras quién eres nunca podrás después cuando quisieres.

Es este cuento del Libro de Patronio o El Conde Lucanor un producto muy de su tiempo. Las colecciones de relatos, ejemplos o apólogos, proliferan durante toda la Edad Media, siguiendo corrientes orientales y alimentándose de temas clásicos o procedentes de la propia tradición indoeuropea. El Conde Lucanor es vivo ejemplo de esta tendencia y primer libro español donde se narran los cuentos ya con un criterio artístico, con una voluntad de estilo, con un afán de perfección no sólo al escoger los relatos sino también en el momento de escribirlos. El esquema estructural del cuento de «El mancebo que casó con una mujer muy fuerte y muy brava» es idéntico a los demás aparecidos en el resto de la colección: el conde Lucanor, ante un problema planteado por el gobierno de su casa, pide consejo a su ayo Patronio, que le responde con un cuento alusivo al problema que tiene que dilucidar. Finalizará el relato con la aplicación al caso y la obtención de una moraleja expresada en un pareado final. Es claro, pues, que el afán didáctico-moral preside la narración del autor, como todas las del libro, aunque en este caso, el tema no sea propiamente moral en abstracto, sino aplicado a la conducta matrimonial ante el caso particular de la mujer brava.

Las preocupaciones de Don Juan Manuel en todo el libro responden a muy diversos asuntos: la vanidad, la hipocresía, la pobreza, los engaños que puede sufrir el ser humano. Son todos sus temas, por tanto, de carácter moral, pero don Juan los trata con singular originalidad, con rasgos muy personales y no es el menor de éstos el manejo de la ironía, y la gracia y la amabilidad marcan la temperatura de casi todos estos apólogos. Tales rasgos artísticos están patentes en este cuento, cuyo tono irónico se ve reflejado en el gusto por la hipérbole, como en los bárbaros sacrificios de los animales, descritos con inusitada plasticidad. E incluso la feliz ironía final dota al relato de indudable gracia, sobre todo en la final alusión al intento de repetir la experiencia por parte del suegro del mancebo. Constituye éste el contrapunto de la acción 
principal del cuento, logrando así completar las posibilidades morales del ejemplo —una positiva, el nuevo esposo; y otra negativa, el suegro- para llegar a la moraleja después de haber ejemplificado los dos extremos de una realidad, moraleja expresada en los dos versos que constituyen el tercer estrato narrativo, representada por la voz del narrador principal, es decir, el propio Don Juan Manuel.

Una cuestión muy interesante, y de la que se ha ocupado una muy importante biografía es la de la tradicionalidad del cuento. Por supuesto, éste se encuentra, con su historia, en colecciones de apólogos, y pertenece al folklore oriental. El origen concreto de esta historia es persa y su resonancia posterior contará nada menos que, entre otras muchas versiones, con una de las mejores comedias de William Shakespeare, The Taming of the Shrew, que hemos traducido al castellano como La doma de la bravía o La fierecilla domada. Pero también es muy interesante, quizá más, la tradicionalidad de los procedimientos y las estructuras narrativas. $Y$ a ello nos vamos a referir brevemente.

Los cuentos empiezan a hacerse presentes en la literatura castellana de la época a mediados del siglo XIII. Es de suponer que en las largas noches del invierno, en las tertulias de las grandes casas, de los campamentos militares, en las reuniones en las plazas de los pueblos, en los viajes, en los descansos en las posadas, se contasen cuentos. Y por ello, no es de extrañar que Alfonso $X$ el Sabio mandase traducir la primera colección importante de cuentos que se conoce en castellano, el libro del Calila e Dimna, ejemplo que fue seguido por su levantisco hermano el infante Don Fadrique, que mandó traducir, como ya sabemos, el Libro de los engaños e assayamientos de las mujeres o Sendebar. Tanto el Calila e Dimna como el Sendebar se caracterizan por contener relatos con estructura repetitiva, como la tiene éste de Don Juan Manuel, del "Mancebo que casó con una mujer muy fuerte e muy brava". En el Sendebar hay un cuento que tiene este mismo efecto de carambola: "Oí decir que un cazador que andaba cazando por el monte, halló en un árbol un enjambre, y tomólo y metiólo en un odre que tenía para traer su agua; y este cazador tenía un perro, y traíalo consigo; y trajo la miel a un mercader de una aldea que era cerca de aquel monte para la vender. Y cuando el cazador abrió el odre para lo mostrar al tendero, cayó de él una gota y posóse en ella una abeja; y aquel tendero tenía un gato, y dio un salto en la abeja y matóla; el perro del cazador dio un salto sobre el gato y lo mató; y vino el dueño del gato y mató al perro; y entonces levantóse el dueño del perro y mató al tendero, porque le matara el perro; y entonces vinieron los de la aldea del tendero y mataron al cazador dueño del perro y vinieron los de la aldea del cazador a los del tendero, y tomáronse unos con otros y matáronse todos, que no fincó y ninguno; y se mataron unos con otros por una gota de miel». En una carta del murciano Alonso Fajardo, ya en siglo XV, se recoge este mismo clímax de horrores o efecto carambola: "Soez cosa es un clavo y por él se pierde una herradura, y por una herradura un caballo, y por un caballo una hueste y por una hueste, una ciudad y un reino» ${ }^{19}$.

19 Sendebar, edición de José Fradejas Lebrero, Editora Nacional, Madrid, 1981, p. 91. 
En el caso del cuento de Don Juan Manuel que ahora comentamos, el diseño repetitivo nos conduce rápidamente al momento clave del relato: aquel en que para dominar a su mujer el marido pide agua para las manos a un perro, a un gato y a un caballo. Es obvio que el movimiento repetitivo podría haberse alargado si Don Juan Manuel se hubiera servido de más seres vivos, de más animales que los tenidos en cuenta: un perro, un gato y un cabalio. Pero con poderoso instinto narrativo, con gran sentido de la proporción y del ritmo del cuento, Don Juan Manuel detiene el gracioso mecanismo repetitivo justamente donde convenía ya que de haberse prolongado la lista, se hubiese descendido en el nivel de eficacia narrativa. Como un eco de ese movimiento repetitivo se nos ofrece, al final del cuento, el sacrificio del gallo por el suegro sin resultado alguno. Se trata, como yo le of explicar a mi maestro el Profesor Mariano Baquero Goyanes ${ }^{20}$, de una especie de festiva coda o cola final que, a la par que introduce una inteligente nota humorística en el relato, refuerza la disposición repetitiva de su estructura.

El genio de Don Juan Manuel, el mismo genio que utilizó para gobernar su Adelantamiento Mayor del Reino de Murcia, le sirvió para configurar el más serio avance que se produce en la prosa literaria medieval en la Península.

20 Aunque lo referido no está en sus páginas, merece consultarse, para cuestiones de narrativa, Mariano Baquero Goyanes, "Perspectivismo en EL Conde Lucanor", Don Juan Manuel VII Centenario, Universidad de Murcia-Academia Alfonso X el Sabio, Murcia, 1982, pp. 27-50. 